\title{
In utero exposure to gestational diabetes mellitus and cardiovascular risk factors in youth: A longitudinal analysis in the EPOCH cohort
}

\author{
Wei Perng $\mathrm{PhD}^{1,2,3}$ (1) | Christine W. Hockett $\mathrm{PhD}^{1}$ ( ) | \\ Katherine A. Sauder PhD ${ }^{1,4}$ - | Dana Dabelea MD, PhD
}

\section{${ }^{1}$ Lifcourse Epidemiology of Adiposity and Diabetes (LEAD) Center, University of Colorado Anschutz Medical Campus, Aurora, Colorado \\ ${ }^{2}$ Department of Epidemiology, Colorado School of Public Health, Aurora, Colorado \\ ${ }^{3}$ Department of Nutritional Sciences, University of Michigan School of Public Health, Ann Arbor, Michigan \\ ${ }^{4}$ Department of Pediatrics, University of Colorado School of Medicine, Aurora, Colorado}

\section{Correspondence}

Wei Perng, Lifecourse Epidemiology of Adiposity and Diabetes (LEAD) Center, University of Colorado Anschutz Medical Campus, Aurora, CO 80045.

Email: wei.perng@ucdenver.edu

Funding information

National Institute of Diabetes and Digestive and Kidney Diseases, Grant/Award Number: R01 DK068001

\section{Summary}

Objective: To examine associations of maternal gestational diabetes mellitus (GDM) with offspring cardiovascular biomarkers from late childhood through adolescence.

Methods: We used mixed effects linear regression models to examine associations of maternal GDM ( $\mathrm{n}=92$ cases of 597) with average offspring levels of serum lipids (total cholesterol, high-density lipoprotein [HDL], low-density lipoprotein [LDL], and triglycerides) and systolic blood pressure (SBP) across two research visits spanning approximately 10.6 and 16.9 years of age. In sex-stratified analysis, we evaluated the impact of adjustment for sociodemographic characteristics, pubertal status, physical activity and total energy intake, maternal body mass index (BMI), GDM treatment, and child's BMI.

Results: After adjusting for child's age, pubertal status, race/ethnicity, and maternal education and smoking, GDM exposure was associated with higher total (0.38 [95\% $\mathrm{Cl}, 0.16-0.61] \mathrm{mmol} / \mathrm{L})$ and $\mathrm{LDL}$ cholesterol $(0.34[95 \% \mathrm{Cl}, 0.14-0.53] \mathrm{mmol} / \mathrm{L})$ in girls. These estimates were robust to adjustment for lifestyle characteristics and maternal BMI but were attenuated after accounting for GDM treatment with no appreciable change following further adjustment for current BMI. In boys, maternal GDM corresponded with 4.50 (1.90-7.10) $\mathrm{mmHg}$ higher SBP. This association persisted after accounting for sociodemographic/lifestyle characteristics, maternal BMI, and GDM treatment but was attenuated after adjusting for current BMI.

Conclusions: Maternal GDM is related to offspring lipid profile and SBP in a sexspecific manner.

\section{KEYWORDS}

adolescence, blood pressure, gestational diabetes mellitus, longitudinal, low-density lipoprotein, total cholesterol
Abbreviations: BMI, body mass index; HDL, high-density lipoprotein; LDL, low-density lipoprotein; METs, metabolic equivalents; MVPA, moderate to vigorous physical activity; SBP, systolic blood pressure.

\section{1 | INTRODUCTION}

In his famous 1980 lecture, "Of Pregnancy and Progeny," Norbert Freinkel called gestational diabetes mellitus (GDM) a "teratogen" that leads to higher birthweight and congenital defects. ${ }^{1} \mathrm{~A}$ number of 
epidemiological studies have since noted detrimental effects of maternal GDM on offspring fat distribution starting at $b_{i r t h}{ }^{2-6}$ and on glycaemic regulation (insulin resistance, impaired glucose tolerance, low insulin secretion, and reciprocally altered adipokines ${ }^{6-9}$ ) by age 6 years. Fewer studies have evaluated associations of in utero exposure to GDM with biomarkers of cardiovascular risk, which are important to consider given the interrelations among cardiovascular and metabolic biomarkers, and the persistent burden of cardiovascular disease worldwide ${ }^{10}$-a nonnegligible proportion of which may be traced back to the gestational metabolic milieu. ${ }^{11}$ The majority of publications that have assessed the relation between maternal GDM and cardiovascular health in offspring focused on the adolescent life stage (although one study was conducted in children aged 3 years ${ }^{12}$ ) and identified associations with higher offspring blood pressure ${ }^{6,12-16}$ and an adverse lipid profile. ${ }^{16}$ Many of these studies were able to establish associations independent of offspring adiposity, ${ }^{6,12,14}$ suggesting a unique influence of maternal GDM on in utero "programming" of cardiovascular risk.

In this prospective study of greater than 500 multi-ethnic youth, we test the hypothesis that in utero exposure to maternal GDM with biomarkers of cardiovascular risk in offspring from late childhood through adolescence in the Exploring Perinatal Outcomes in Children (EPOCH) cohort. The biomarkers of interest included serum lipid profile (total cholesterol, triglycerides, high-density lipoprotein [HDL], and low-density lipoprotein [LDL]) and blood pressure, which track from childhood/adolescence into adulthood and are independent predictors of future cardiovascular and metabolic disease risk. ${ }^{17-21}$ This work extends current knowledge in this area of research in two key ways. First, we investigate associations of maternal GDM with biomarkers of cardiovascular risk longitudinally across a period that spans the adolescent transition via a repeated measures approach. Given that multiple measurements of health are more informative than single measurements, our analytical strategy is an improvement upon that of current literature, which has focused on evaluating associations of early life exposures with health outcomes at a single point in time later in life (including an earlier study in $\mathrm{EPOCH}$ that focused on data at the first research visit only ${ }^{6}$ ). Second, in all models, we consider sex-specific associations given documented differences in fetal (as well as later-life ${ }^{2}$ ) response to in utero exposures ${ }^{22-24}$ and sex differences in metabolism during the adolescent life stage. ${ }^{25}$

\section{2 | METHODS}

\section{1 | Study population}

Study participants were from a historical prospective cohort of youth whose mothers were members of the Kaiser Permanente of Colorado (KPCO) Health plan. Details on eligibility and recruitment are published. ${ }^{3}$ Of the original 604 participants enrolled, we excluded from the present analysis children of seven women with type 1 diabetes. The analytic sample comprised 597 youth at the first research visit and 410 at the second one with information on at least one cardiovascular risk factor of interest. The Colorado Multiple Institutional Review Board approved this study.

\section{2 | Exposure}

All pregnant women at KPCO are routinely screened for GDM at 24 to 28 weeks using the standard two-step protocol. ${ }^{26} \mathrm{~A}$ total of 92 women in the sample had a GDM diagnosis.

\section{3 | Outcomes}

Using blood collected after an 8-hour fast, total cholesterol, HDL, and triglycerides were assayed on the Olympus AU400 advanced chemistry analyser system. We calculated LDL as (total cholesterol) - (HDL cholesterol) - (triglycerides/5). Research assistants measured participants' blood pressure twice in the sitting position using an oscillometric monitor (Dinamap ProCare V100). We used the average of the two values in the analysis and focused on systolic blood pressure (SBP) rather than diastolic blood pressure because it is more accurately measured in children and is a stronger determinant of future health outcomes. ${ }^{27}$

\subsection{Covariates}

\subsection{1 | Perinatal and delivery characteristics}

We calculated maternal pre-pregnancy body mass index (BMI; $\mathrm{kg} / \mathrm{m}^{2}$ ) using clinically recorded pre-pregnancy weight from KPCO medical records and measured height at the first research visit. The medical record also provided information on pregnancy complications such as preeclampsia and birthweight (g) as well as delivery characteristics like gestational age at delivery (weeks) for the index birth.

At T1, the women reported on smoking habits during pregnancy, education level at time of birth, and total gestational weight gain (lbs) via a self-administered questionnaire. In the analysis, we categorized maternal education as a three-level variable ("<high school," "high school diploma or equivalent," and ">high school") and smoking as a dichotomous variable (smoked while pregnant with index child, yes vs no). At this visit, the mothers also filled out a questionnaire regarding any treatment they received for GDM. GDM treatment was categorized as diet and/or exercise only, diet and/or exercise with insulin, and insulin only. Because of the small sample size for insulin only treatment $(n=5)$, we combined this category with diet and/or exercise when using this variable as a covariate in the analysis.

\subsection{2 | Participant characteristics}

We calculated participants' age as the difference between date of each research visit and delivery date. At both visits, we measured the 
participants' weight on a digital scale to the nearest $0.1 \mathrm{~kg}$ and measured height $(\mathrm{cm})$ via a calibrated stadiometer to the nearest $0.1 \mathrm{~m}$. We calculated BMI and standardized it according to the age- and sexspecific World Health Organization (WHO) growth reference. ${ }^{28}$ Participants self-reported on their race/ethnicity as non-Hispanic White, non-Hispanic Black, Hispanic, and non-Hispanic other. Participants reported their pubertal development based on diagrams of the Tanner stages. We based pubertal status on pubic hair development in boys and breast development in girls. For each characteristic, we classified a child as pre-pubertal (Tanner stage $=1$ ) or pubertal (Tanner stag$e \geq 2$ ). We obtained information on the participants' physical activity levels using the 3-Day Physical Activity Recall (3DPAR) Questionnaire. ${ }^{29}$ Using 3DPAR, we derived average energy expenditure (metabolic equivalents; METs) and mean number of moderate- to-vigorous 30-minute blocks of physical activity (METs) over the 3-day period. At both visits, participants completed the Block Kid's Food Frequency Questionnaire (FFQ). ${ }^{30}$ We used these data in conjunction with the USDA Food Composition Database to estimate total energy intake (kcals/day).

\subsection{Data analysis}

First, we assessed bivariate associations of in utero GDM exposure with maternal, perinatal, and offspring characteristics to identify potential confounders. This step, in conjunction with our a priori knowledge of determinants of cardiovascular health in youth, informed our multivariable analysis.

Next, we examined associations of GDM exposure with cardiovascular biomarkers from late childhood through adolescence using a mixed effects linear regression model wherein the outcome of interest was repeated measurements of each biomarker. The explanatory variables included longitudinal assessments of age at each research visit, a random effect for individual ID to account for the repeated outcomes, and an unstructured covariance matrix. We have previously used this approach as a way to characterize growth trajectories from longitudinal assessments of anthropometric data. ${ }^{31-33}$ A key advantage of this approach (as opposed to conventional linear regression to examine associations of an exposure with the outcome of interest at separate time points, or with change in the outcome between specific time points) is that it does not require that all participants have the same number of measurements assessed at exactly the same time. In this study, the participants may have a maximum of two measurements for each biomarker, but if a participant has biomarker data at one visit only, their information still contributes to estimation of standard errors. This approach most efficiently leverages our longitudinal data, both from an analytical standpoint and from the perspective of interpreting the biological relevance of results given that multiple assessments of health are likely more meaningful than single evaluations. Using this approach, the estimate of interest ( $\beta$ for GDM exposure) is interpreted as the association of GDM with average levels of a biomarker over the 6 years of follow-up. A key assumption of using mixed effects models to generate population-level estimates is homogeneity of associations in the face of variation in the age distribution of the population. Thus, we tested for birth cohort effects by including an interaction term between age at first research visit and GDM exposure; the interaction terms provided no evidence of birth cohort effects.

In multivariable analysis, we evaluated the impact of covariate adjustment on the $\beta$ and $95 \%$ confidence interval $(\mathrm{Cl})$ for maternal GDM. Covariates for model 1 included longitudinal assessments of the child's age in years and pubertal status (ie, up to two data points for each variable per child, measured at the same time as the cardiovascular outcomes), as well as race/ethnicity, maternal education at the time of birth, and smoking habits during pregnancy. Model 2 further accounted for longitudinal assessments of mean energy expenditure and total energy intake. Model 3 included model 1 covariates plus maternal pre-pregnancy BMI, given that a woman's pre-gravid weight status likely influences offspring health through shared pathways with hyperglycaemia. Model 4 included model 1 covariates plus GDM treatment to account for variability in maternal hyperglycaemia following a diagnosis of GDM. In Model 5, we adjusted for model 4 covariates plus the child's current BMI to assess for an independent association of maternal GDM (while adjusting for treatment) with offspring cardiovascular biomarkers independent of offspring adiposity.

In all models, we tested for interactions with sex (which tests for a difference in association of GDM with cardiovascular outcomes for girls vs boys), pubertal status at the first visit (which tests for a difference in association of GDM with cardiovascular outcomes by pubertal status), and repeated measurements of age across the two research visit (which tests for differences in slopes of biomarker trajectories over time for GDM exposed vs unexposed). We considered $P$ interaction $<.05$ as the threshold for a need to conduct stratified analysis. As expected, we found evidence of effect modification of association between GDM and cardiovascular risk factors by sex, so we conducted all models separately for boys and girls. We did not observe evidence of effect modification by the other abovementioned variables.

\subsection{1 | Sensitivity analyses}

In addition to the main analysis, we carried out some sensitivity analyses. First, because height is a strong determinant of BP in children and adolescents ${ }^{34}$ and because clinical assessments of paediatric blood pressure are based on age-, sex-, and height-specific values, ${ }^{35}$ we reran models for blood pressure with SBP $z$ score as the outcome. Results for SBP and SBP $z$ score were similar, so we focus on interpretation of SBP in $\mathrm{mmHg}$ for interpretability. Second, given the cooccurrence of GDM and preeclampsia, ${ }^{36}$ we reran all models after excluding 27 mother-offspring pairs affected by preeclampsia. Exclusion of these individuals did not appreciably change our findings; thus, we included these participants in our analysis. Finally, we assessed the impact of adjustment for birthweight and gestational age at delivery, two mediating perinatal characteristics that are determinants of longterm cardiovascular health. ${ }^{37-39}$ Adjusting for these variables did not 
change the direction, magnitude, or precision of the results, so we did not include them in the final models.

We carried out all analyses using Statistical Analyses System software (version 9.3; SAS Institute Inc., Cary, NC).

\section{3 | RESULTS}

Mean $\pm S D$ age of participants at the first research visit was 10.4 \pm 1.5 years (range: 6.0-13.9 years) and $16.7 \pm 1.2$ years (range:
12.6-19.6 years) at the second research visit, with an approximate follow-up time of 6 years. Fifty percent of the participants were female, and $48.1 \%(n=287$ ) were non-Hispanic White. Approximately $15 \%$ ( $n=92$ ) of participants were exposed to GDM in utero. Table 1 shows descriptive characteristics for mother-child dyads stratified by GDM exposure, and Table S1 shows the same characteristics stratified by sex. Associations of GDM exposure status with background characteristics were similar across sex, although we noted that a higher proportion of exposed girls were post-pubertal at the first visit (53.5\% post-pubertal) then exposed boys (24.5\% post-pubertal).

TAB LE 1 Bivariate associations of in utero GDM exposure with background characteristics of 597 EPOCH participants

\begin{tabular}{|c|c|c|c|}
\hline & $\frac{\text { GDM exposed }}{n=92}$ & $\frac{\text { Unexposed }}{n=505}$ & $P$ value* \\
\hline \multicolumn{4}{|l|}{ Maternal characteristics } \\
\hline Maternal education level & & & .50 \\
\hline$<$ High school & $5.4 \%$ & $3.4 \%$ & \\
\hline >High school & $79.4 \%$ & $78.2 \%$ & \\
\hline Mother smoked during pregnancy & $14.1 \%$ & $7.3 \%$ & .03 \\
\hline \multicolumn{4}{|l|}{ GDM treatment } \\
\hline Diet and/or exercise & $66.3 \%$ & - & \\
\hline Yes & $6.5 \%$ & $4.2 \%$ & \\
\hline No & $93.5 \%$ & $95.8 \%$ & \\
\hline \multicolumn{4}{|l|}{ Child's characteristics } \\
\hline Female & $46.70 \%$ & $50.90 \%$ & .46 \\
\hline Race/ethnicity & & & .02 \\
\hline Non-Hispanic White & $63.0 \%$ & $45.4 \%$ & \\
\hline Hispanic & $28.3 \%$ & $40.0 \%$ & \\
\hline Non-Hispanic Black & $4.4 \%$ & $8.5 \%$ & \\
\hline Non-Hispanic other & $4.4 \%$ & $6.1 \%$ & \\
\hline \multicolumn{4}{|l|}{ Anthropometry and lifestyle at T2 } \\
\hline BMI z score & $0.54 \pm 1.05$ & $0.38 \pm 1.13$ & .26 \\
\hline Post-pubertal (Tanner stage $\geq 2$ ) & $100.00 \%$ & $100.00 \%$ & - \\
\hline Mean energy expenditure (METs) & $1.85 \pm 0.27$ & $1.91 \pm 0.32$ & .12 \\
\hline Mean moderate-to-vigorous physical activity (METs) & $4.81 \pm 4.48$ & $4.70 \pm 3.77$ & .82 \\
\hline Total energy intake (kcal/d) & $1683 \pm 767$ & $1653 \pm 725$ & .76 \\
\hline
\end{tabular}

Abbreviations: BMI, body mass index; EPOCH, Exploring Perinatal Outcomes in Children; GDM, gestational diabetes mellitus; MET, metabolic equivalent. *From an independent $t$ test for continuous variables; from a Pearson chi-squared test for categorical variables. 
TAB LE 2 Descriptive statistics for cardiovascular risk factors of $597 \mathrm{EPOCH}$ participants at two research visits (ages 6-14 and 12-19 y)

\begin{tabular}{|c|c|c|}
\hline & Visit 1 & Visit 2 \\
\hline & $n=300$ & $n=206$ \\
\hline \multicolumn{3}{|l|}{ Girls } \\
\hline Age (years) & $10.4 \pm 1.4$ & $16.7 \pm 1.2$ \\
\hline Cholesterol (mmol/L) & $4.1 \pm 0.7$ & $3.8 \pm 0.8$ \\
\hline $\mathrm{HDL}(\mathrm{mmol} / \mathrm{L})$ & $1.3 \pm 0.3$ & $1.2 \pm 0.3$ \\
\hline $\mathrm{LDL}(\mathrm{mmol} / \mathrm{L})$ & $2.3 \pm 0.6$ & $2.1 \pm 0.6$ \\
\hline Triglycerides (mmol/L) & $1.1 \pm 0.5$ & $1.0 \pm 0.6$ \\
\hline $\mathrm{SBP}(\mathrm{mmHg})$ & $102.1 \pm 9.6$ & $112.0 \pm 9.7$ \\
\hline Boys & $n=297$ & $n=204$ \\
\hline Age (years) & $10.5 \pm 1.5$ & $16.6 \pm 1.1$ \\
\hline Cholesterol (mmol/L) & $4.1 \pm 0.8$ & $3.6 \pm 0.6$ \\
\hline $\mathrm{HDL}(\mathrm{mmol} / \mathrm{L})$ & $1.3 \pm 0.3$ & $1.1 \pm 0.2$ \\
\hline LDL (mmol/L) & $2.4 \pm 0.7$ & $2.0 \pm 0.5$ \\
\hline Triglycerides (mmol/L) & $1.0 \pm 0.4$ & $1.0 \pm 0.4$ \\
\hline $\mathrm{SBP}(\mathrm{mmHg})$ & $103.8 \pm 10.3$ & $119.4 \pm 10.6$ \\
\hline
\end{tabular}

Abbreviations: EPOCH, Exploring Perinatal Outcomes in Children; HDL, high-density lipoprotein; LDL, low-density lipoprotein; SBP, systolic blood pressure.
Table 2 shows mean $\pm S D$ for the cardiovascular risk biomarkers of interest at each visit for all children and separately by sex, respectively.

Table 3 displays sex-specific associations of GDM exposure with cardiovascular risk factors over the entire age-range of study participants from both research visits. We interpret these results as the association of in utero GDM exposure on average levels of each cardiovascular risk factor across the two research visits (rather than average trajectories of change per year) as we did not find a significant interaction between GDM exposure and time, which suggests a consistent influence of GDM exposure on these health outcomes. After accounting for key confounders in model 1 , girls who were exposed to GDM in utero had $0.38(95 \% \mathrm{Cl}, 0.16-0.61) \mathrm{mmol} / \mathrm{L}$ and $0.34(95 \%$ $\mathrm{Cl}, 0.14-0.53) \mathrm{mmol} / \mathrm{L}$ higher total cholesterol and LDL, respectively, than their unexposed counterparts. This association was robust to adjustment for physical activity and total energy intake (model 2) and maternal pre-pregnancy BMI (model 3). However, adjustment for GDM treatment in model 4 attenuated both estimates to approximately $2 / 3$ the magnitude, with the lower $\mathrm{Cl}$ crossing the null (Table 3). Further adjustment for the offspring's current BMI $z$ score in model 5 did not change the estimates.

In boys, GDM exposure corresponded with $4.50(95 \% \mathrm{Cl}$, 1.90-7.10) $\mathrm{mmHg}$ higher SBP from late childhood through adolescence

TABLE 3 Associations of in utero exposure to GDM with CV traits across in 597 EPOCH participants across T1 (age 6-14 y) and T2 (age $12-19$ y)

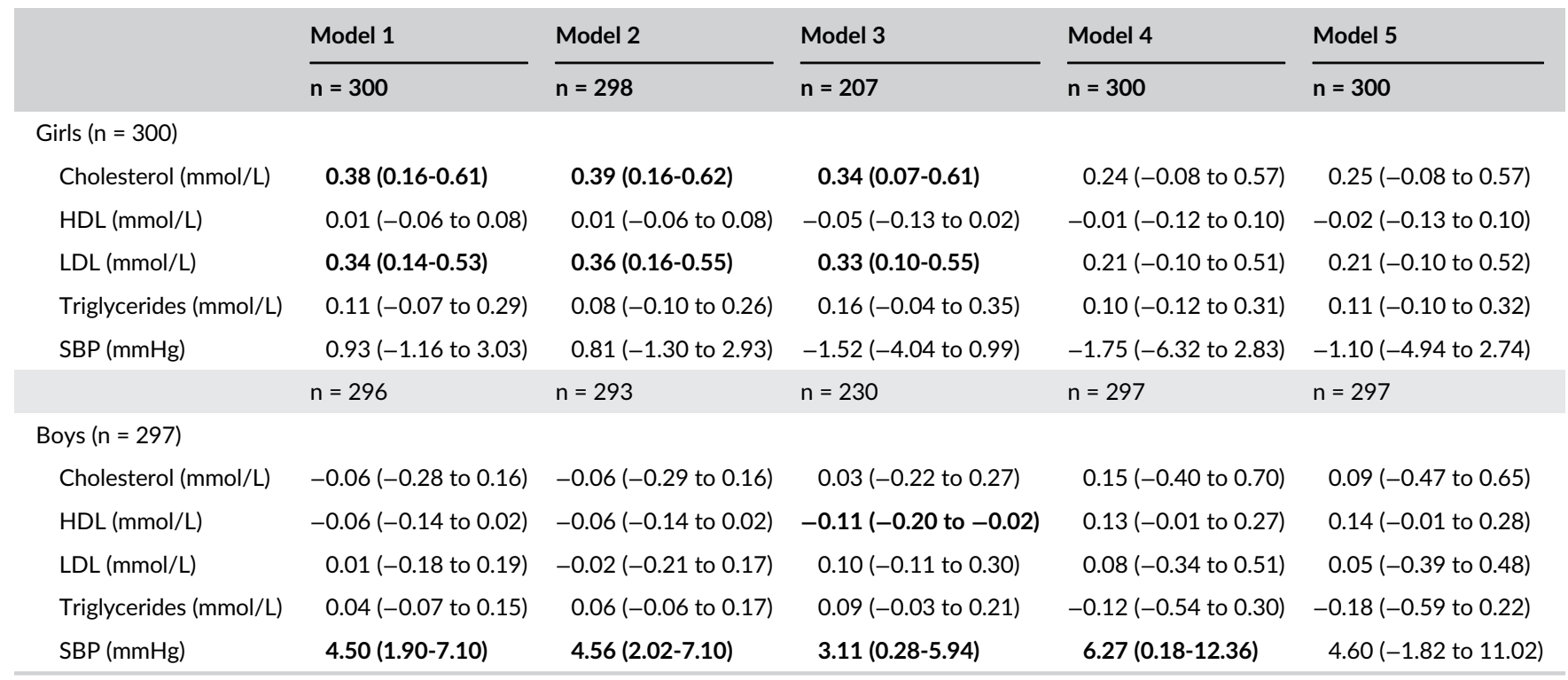

Bold face font indicates statistical significance at $\alpha=0.05$.

Notes: Estimates are from a linear mixed effects model where the dependent variable is repeated measurements of the CVD traits across T1 and T2, and the predictors include GDM (yes vs no), covariates, and a random effect for individual intercept.

Model 1: Adjusted for child's age (years), pubertal status (pre vs post-pubertal), race/ethnicity (non-Hispanic White, Hispanic, non-Hispanic Black, and non-Hispanic other), maternal smoking habits during pregnancy (yes vs no) and education level at time of birth (<high school, high school, and $>$ high school).

Model 2: Model $1+$ physical activity levels (average energy expenditure; METs) and total energy intake (kcals).

Model 3: Model $1+$ maternal pre-pregnancy BMI.

Model 4: Model $1+$ GDM treatment (diet/exercise only vs none, diet/exercise with insulin vs none).

Model 5: Model $4+$ child's BMI z score.

Abbreviations: CV, cardiovascular; EPOCH, Exploring Perinatal Outcomes in Children; GDM, gestational diabetes mellitus; HDL, high-density lipoprotein; LDL, low-density lipoprotein; SBP, systolic blood pressure. 


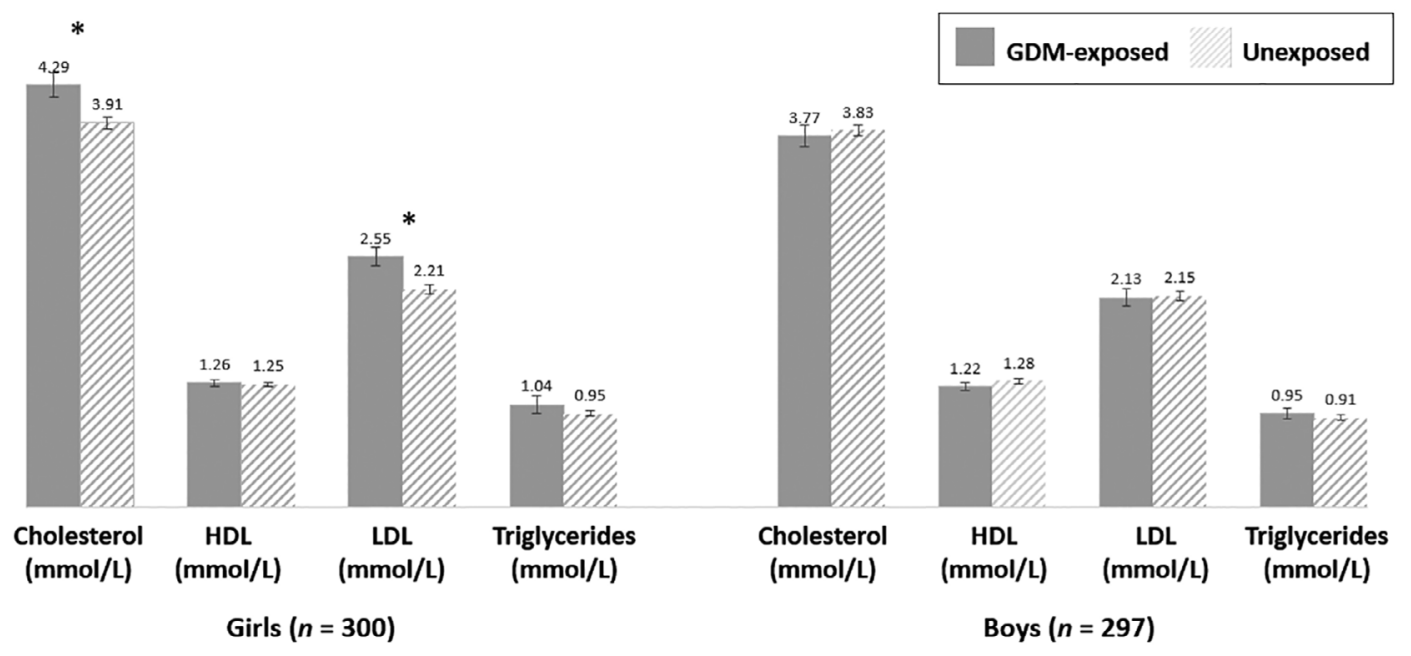

FIGURE 1 Mean \pm SE for lipid profile components among GDM-exposed vs unexposed EPOCH participants. Estimates are adjusted for child's age (years), pubertal status (pre- vs post-pubertal), race/ethnicity (non-Hispanic White, Hispanic, non-Hispanic Black, and non-Hispanic other), maternal smoking habits during pregnancy (yes vs no), and education level at time of birth (<high school, high school, and >high school). *Statistically significant difference at $\alpha=.05$. EPOCH, Exploring Perinatal Outcomes in Children; GDM, gestational diabetes mellitus; HDL, highdensity lipoprotein; LDL, low-density lipoprotein

GDM-exposed Unexposed

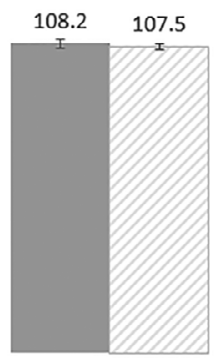

SBP ( $\mathrm{mmHg})$

Girls $(n=300)$

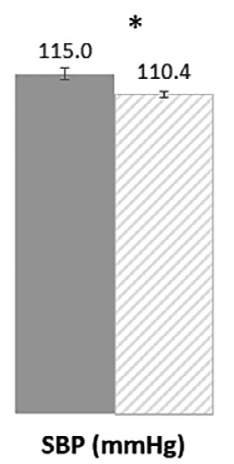

Boys $(n=297)$
FIGURE 2 Mean \pm SE for SBP among GDM-exposed vs unexposed EPOCH participants. Estimates are adjusted for child's age (years), pubertal status (pre- vs post-pubertal), race/ethnicity (nonHispanic White, Hispanic, non-Hispanic Black, and non-Hispanic other), maternal smoking habits during pregnancy (yes vs no) and education level at time of birth (<high school, high school, and >high school). *Statistically significant difference at $\alpha=.05$. EPOCH, Exploring Perinatal Outcomes in Children; GDM, gestational diabetes mellitus; HDL, high-density lipoprotein; LDL, low-density lipoprotein; $\mathrm{SBP}$, systolic blood pressure

(Table 3, model 1). Accounting for physical activity and total energy intake in model 2 did not change this estimate, although adjusting for maternal pre-pregnancy BMI in model 3 attenuated the estimate by approximately $30 \%$. In model 4 , adjustment for GDM treatment augmented the association to $6.27(95 \% \mathrm{Cl}, 0.18-12.36) \mathrm{mmHg}$ for exposed vs unexposed males. However, additional adjustment for the offspring's current BMI $z$ score attenuated the estimate such that the magnitude was similar to those detected in models 1 and 2 , and the lower $\mathrm{Cl}$ crossed the null $(4.60$ [95\% $\mathrm{Cl},-1.82$ to 11.02$] \mathrm{mmHg}$ ). We also noted that GDM exposure was associated with $0.11(95 \% \mathrm{Cl}$, 0.02-0.20) $\mathrm{mmol} / \mathrm{L}$ lower HDL in boys, although the $95 \% \mathrm{Cl}$ included the null for all models except Model 3.

Table S2 presents estimates of mean $\pm S E$ for the cardiovascular risk factors with respect to GDM exposure after confounder adjustment (model 1). We provide graphical depictions of these results in Figure 1 (lipid profile) and Figure 2 (blood pressure).

Table S3 shows the same set of models as in Table 3 for sex-, age-, and height-specific blood pressure $z$ scores. The results from analyses of $z$ scores are similar to those of blood pressure in $\mathrm{mmHg}$, indicating no concern regarding residual confounding by age or height in our primary analysis.

\section{4 | DISCUSSION}

In this prospective study of 597 multi-ethnic youth in Colorado, we found sex-specific associations of in utero exposure to GDM with lipid profile and blood pressure in offspring from late childhood through adolescence. In girls, GDM was associated with higher total cholesterol and LDL. In boys, GDM corresponded with consistently higher SBP. These associations were consistent across the age-range studied and did not change over time.

\section{1 | Girls}

In utero exposure to GDM corresponded with an average of $0.38 \mathrm{mmol} / \mathrm{L}(6.8 \mathrm{mg} / \mathrm{dL})$ higher total cholesterol, and $0.34 \mathrm{mmol} / \mathrm{L}$ 
(6.1 mg/dL) higher LDL cholesterol levels. These findings are comparable with those of an analysis of 96 male and female Polish youth aged 7 to 16 years wherein Wilk et al ${ }^{40}$ detected unadjusted differences of 19.0 and $14.2 \mathrm{mg} / \mathrm{dL}$ higher total and LDL cholesterol, respectively, among GDM-exposed $(n=50)$ vs unexposed participants $(n=46)$. In a recent study of greater than 1000 boys and girls in the Danish National Birth Cohort, Grunnet et al reported higher serum triglycerides $(5 \%$ [95\% Cl, 1-10\%]) and lower HDL $(-0.07$ [95\% Cl, -0.11 to $-0.02] \mathrm{mmol} / \mathrm{L})$, as well as marginally higher $\mathrm{LDL}(0.06[95 \% \mathrm{Cl},-0.01$ to $0.14 \mathrm{mmol} / \mathrm{L}$ ) at 12 years of age among GDM-exposed youth. ${ }^{16}$ However, these associations became null after adjusting for maternal and child BMI.

In the present analysis, the influence of maternal GDM on the above offspring cardiovascular risk factors remained apparent after accounting for offspring characteristics known to impact lipid profile, including pubertal status, physical activity, and energy intake, as well as maternal pre-pregnancy BMI, a proxy for maternal adiposity that is hypothesized to account for a portion of variability in the relationship between maternal hyperglycaemia and offspring health. However, accounting for GDM treatment attenuated the estimates for cholesterol and LDL towards the null, thereby providing support for a specific effect of maternal hyperglycaemia on these cardiovascular traits. In other words, if removing variability in maternal hyperglycaemia during gestation by adjusting for GDM treatment in the analysis resulted in smaller and nonsignificant effect estimates for maternal GDM, then maternal hyperglycaemia is likely a determinant of the offspring health outcomes.

Our finding that exposure to maternal GDM was associated with an adverse lipid profile that is likely related to the occurrence of perturbed maternal lipid metabolism in the context of gestational hyperglycaemia, ${ }^{41}$ which in turn, may affect offspring lipid metabolism. ${ }^{42}$ Mechanistic pathways are difficult to disentangle in humans, but animal models indicate an impact of maternal hyperglycaemia on hepatic lipid content and metabolism through oxidative stress and inflammatory pathways. ${ }^{43}$ Although we adjusted for pubertal status in multivariable analyses, the female-specific nature of the relationship between exposure to maternal GDM and offspring lipid profile may be related to unmeasured confounding by tempo of sexual maturation (which exists on a spectrum that is likely not wholly captured by discrete Tanner staging) given recent findings that GDM-exposed girls undergo puberty earlier than their male counterparts. Earlier puberty may in turn translate to more rapid increases in total cholesterol and LDL. $^{44}$

\section{2 | Boys}

Boys who were exposed to GDM in utero had approximately $5 \mathrm{mmHg}$ higher SBP than their unexposed counterparts. This relationship remained apparent after accounting for sociodemographic and lifestyle characteristics during follow-up but was modestly attenuated after accounting for maternal pre-pregnancy BMI and current BMI. Interestingly, adjustment for GDM treatment did not attenuate the estimate, but rather, slightly increased the magnitude of effect of maternal GDM from approximately $5 \mathrm{mmHg}$ (models 1 and 2) to approximately $6 \mathrm{mmHg}$ higher SBP for GDM-exposed vs unexposed boys (model 4). This indicates the existence of mechanisms other than hyperglycaemia linking in utero GDM exposure to offspring blood pressure. Indeed, when we further adjusted for the offspring's current BMI $z$ score (in addition to GDM treatment), the estimate for maternal GDM in relation to SBP was approximately $5 \mathrm{mmHg}$ and attenuated to nonsignificance, suggesting that the relationship between maternal hyperglycaemia and offspring SBP is mediated by the offspring adiposity.

In a previous study conducted in this cohort that examined consequences of maternal GDM on adiposity and cardiometabolic biomarkers at the first visit, West et $\mathrm{al}^{6}$ detected a marginally significant $2 \mathrm{mmHg}$ higher SBP between GDM-exposed and unexposed participants at 6 to 13 years. At this time, the investigators reported no differences in the relationship between GDM and health outcomes by sex and adjustment for maternal and child BMI attenuated the associations to the null. Similarly, in an investigation of 1238 children in the Project Viva cohort, Wright et $\mathrm{al}^{12}$ found that GDM exposure corresponded with $3.2 \mathrm{mmHg}$ higher SBP at 3 years of age after adjusting for key covariates including for maternal pre-pregnancy BMI. As with the study of West et al, further adjustment for the chiId's current BMI markedly attenuated the estimate.

In addition to investigations that focused exclusively on GDM, smaller studies have explored offspring cardiovascular consequences of exposure to maternal diabetes during pregnancy based on a combination of pre-existing diabetes (type 1 or type 2) and GDM with offspring blood pressure. Cho et $\mathrm{al}^{15}$ noted $8 \mathrm{mmHg}$ higher SBP $(P<.001)$ in a multi-ethnic population of 99 offspring aged 10 to 16 years, as compared with 80 unexposed counterparts after controlling for height, weight, sex, age, and pubertal status. Similarly, Rostand et al ${ }^{13}$ reported $8 \mathrm{mmHg}(P<.05)$ higher SBP among Black and White children born to diabetic mothers as compared with those of nondiabetic mothers ( $n=10$ diabetic vs 252 nondiabetic) after accounting for sex, child's height, and maternal socio-economic factors. Finally, in the Pima Indian Study, Bunt et al ${ }^{14}$ investigated associations of maternal diabetes with offspring cardiovascular traits after accounting for \% fat mass and sex and detected $11 \mathrm{mmHg}$ higher SBP among 22 Pima Indian children aged 7 to 11 years exposed to diabetes in utero, as compared with 20 offspring of nondiabetic women. Yet again, these studies did not assess the impact of any treatments or interventions to address hyperglycaemia during pregnancy.

Taken together, the current body of evidence suggestions that maternal GDM is associated with higher blood pressure in offspring. While the underlying physiological mechanisms are not known, our data and those of others ${ }^{12}$ suggest that this relationship may be mediated by excess adiposity given the established influence of maternal hyperglycaemia on offspring fat mass at birth and beyond. ${ }^{2-6}$ Animal studies also suggest the possibility of a direct effect of hyperglycaemia on kidney structure and function, ${ }^{45}$ which can affect blood pressure. While this hypothesis has not specifically been tested in humans, a study in the Pima Indians found that exposure to maternal 
diabetes corresponded with nearly four times higher urinary albumin excretion in the offspring, pointing towards the capacity of maternal diabetes to affect fetal nephron development. ${ }^{46}$ Another possible mechanism linking maternal GDM to an adverse lipid profile involves the co-occurrence of maternal dyslipidaemia, which may influence offspring blood pressure through other mechanisms. ${ }^{15}$ The fact that we detected associations of GDM with SBP in boys but not girls may be related to the more rapid increase and greater variability in SBP from late childhood through adolescence in males than females. ${ }^{25}$

\subsubsection{Strengths and limitations}

Our study had several strengths. First, we were able to examine longterm consequences of exposure to maternal GDM on offspring cardiovascular risk factors across the adolescent transition, a sensitive period for development of cardiometabolic disease. ${ }^{47}$ Second, rich covariate data enabled us to adjust for variables that may confound or mediate associations of interest. Third, our longitudinal modelling strategy appropriately accounted for correlations among repeated assessments of cardiovascular risk factors and efficiently leveraged the outcome data across two study visits spanning approximately 6 years of follow-up.

However, this study is not without limitations. A weakness of this study is the fact that we only had access to medical record data that provided information on GDM diagnosis, thereby precluding our ability to explore associations of the in utero glycaemic milieu at a finer resolution. A second limitation is the slightly decreasing sample sizes in multivariable models because of missing data for covariates, although the subsamples did not differ with respect to sociodemographic characteristics. A third limitation is the potential lack of generalizability because of over-selection of participants exposed to maternal GDM. Finally, we cannot discount the potential for false positive findings given the large number of models. However, our research focus was to describe and assess the direction, magnitude, and precision of the estimates rather than focus on statistical significance, especially in light of the fact that many of the biomarkers are correlated markers of the same biological phenomena.

\section{5 | CONCLUSIONS}

In this prospective study of approximately 600 multi-ethnic youth, we extend current literature regarding the relationship between in utero GDM exposure and cardiovascular health in offspring in two key aspects. First, we were able to explore the long-term consequences of maternal GDM on offspring cardiovascular risk factors throughout late childhood and adolescence via a repeated measures technique. This approach is not only more efficient from a statistical standpoint but also lends itself to more robust findings given that the biomarkers of interest-including total cholesterol and LDL, which we found to differ by GDM exposure in females-fluctuate across the age range of our study sample. ${ }^{44}$ Second, we considered sex-specific associations to reflect not only biological differences metabolic and cardiovascular traits as youth progress through the adolescent transition ${ }^{25}$ but also potential discrepancies in fetal response to in utero exposures like maternal glycaemic control. ${ }^{2}$

While the effect sizes that we detected are modest (approximately $0.35 \mathrm{mmol} / \mathrm{L}$ higher total cholesterol and $0.31 \mathrm{mmol} / \mathrm{L}$ higher $\mathrm{LDL}$ in girls; approximately $5 \mathrm{mmHg}$ higher SBP in boys for GDM exposed vs unexposed), they may have important long-term ramifications given that cardiometabolic risk factors track from childhood/adolescence into adulthood and are independent predictors of cardiovascular disease risk. ${ }^{48}$ For example, in the Bogalusa Heart Study, a 1-mg/dL increment in LDL (equivalent to an increment of $0.026-\mathrm{mmol} / \mathrm{L} \mathrm{LDL}$ ) at 11 years of age corresponded with nearly two times higher odds of metabolic syndrome in young adulthood. ${ }^{49}$ Similarly, a 1-mmHg increment in childhood SBP predicted elevations in a cluster of metabolic risk factors in young adulthood including SBP, cholesterol, triglycerides, and fasting glucose among Bogalusa Heart Study participants. ${ }^{21}$ Given the increasing prevalence of GDM in developed countries like the United States, our findings underscore the importance of efforts to prevent maternal diabetes in order to improve the health of future generations.

\section{ACKNOWLEDGEMENTS}

This study was supported by the National Institutes of Health (NIH), National Institute of Diabetes, Digestive, and Kidney Diseases (R01 DK068001). The funders had no role in the design, conduct, or reporting of this work. We are grateful for Brandy M. Ringham for data preparation and Deborah Glueck for analytical support. We thank the EPOCH participants, as well as past and present research assistants.

\section{CONFLICT OF INTEREST}

The authors have no conflicts of interest to report.

\section{AUTHOR CONTRIBUTIONS}

WP and DD conceived research questions. WP conducted the analysis, wrote the initial draft of the paper, and incorporated co-author comments. CWH and KS contributed to the analytical approach. All authors were involved in writing the paper and approved the final version.

\section{ORCID}

Wei Perng (D) https://orcid.org/0000-0001-8552-6850

Christine W. Hockett (D) https://orcid.org/0000-0002-1665-6763

Katherine A. Sauder (D) https://orcid.org/0000-0002-8473-8015

\section{REFERENCES}

1. Freinkel N. Banting lecture 1980. Of pregnancy and progeny. Diabetes. 1980;29(12):1023-1035.

2. Regnault N, Gillman MW, Rifas-Shiman SL, Eggleston E, Oken E. Sexspecific associations of gestational glucose tolerance with childhood body composition. Diabetes Care. 2013;36(10):3045-3053.

3. Crume TL, Ogden L, West NA, et al. Association of exposure to diabetes in utero with adiposity and fat distribution in a multiethnic 
population of youth: the exploring perinatal outcomes among children (EPOCH) study. Diabetologia. 2011;54(1):87-92.

4. Chang $\mathrm{Y}$, Chen $\mathrm{X}$, Zhang ZK. Intrauterine exposure to maternal diabetes is associated with adiposity in children at 6 years of age in China. Biomed Environ Sci. 2015;28(2):140-142.

5. Kc K, Shakya S, Zhang H. Gestational diabetes mellitus and macrosomia: a literature review. Ann Nutr Metab. 2015;66(Suppl 2): 14-20.

6. West NA, Crume TL, Maligie MA, Dabelea D. Cardiovascular risk factors in children exposed to maternal diabetes in utero. Diabetologia. 2011;54(3):504-507.

7. Sauder KA, Hockett CW, Ringham BM, Glueck DH, Dabelea D. Fetal overnutrition and offspring insulin resistance and beta-cell function: the exploring perinatal outcomes among children (EPOCH) study. Diabet Med. 2017;34(10):1392-1399.

8. Krishnaveni GV, Veena SR, Hill JC, Kehoe S, Karat SC, Fall CH. Intrauterine exposure to maternal diabetes is associated with higher adiposity and insulin resistance and clustering of cardiovascular risk markers in Indian children. Diabetes Care. 2010;33(2):402-404.

9. Gautier JF, Wilson C, Weyer C, et al. Low acute insulin secretory responses in adult offspring of people with early onset type 2 diabetes. Diabetes. 2001;50(8):1828-1833.

10. Roth GA, Johnson C, Abajobir A, et al. Global, regional, and national burden of cardiovascular diseases for 10 causes, 1990 to 2015. J Am Coll Cardiol. 2017;70(1):1-25.

11. Oken E, Gillman MW. Fetal origins of obesity. Obes Res. 2003;11(4): 496-506.

12. Wright CS, Rifas-Shiman SL, Rich-Edwards JW, Taveras EM, Gillman MW, Oken E. Intrauterine exposure to gestational diabetes, child adiposity, and blood pressure. Am J Hypertens. 2009;22(2): 215-220.

13. Rostand SG, Cliver SP, Goldenberg RL. Racial disparities in the association of foetal growth retardation to childhood blood pressure. Nephrol Dial Transplant. 2005;20(8):1592-1597.

14. Bunt JC, Tataranni PA, Salbe AD. Intrauterine exposure to diabetes is a determinant of hemoglobin $\mathrm{a}(1) \mathrm{c}$ and systolic blood pressure in pima Indian children. J Clin Endocrinol Metab. 2005;90(6):3225-3229.

15. Cho NH, Silverman BL, Rizzo TA, Metzger BE. Correlations between the intrauterine metabolic environment and blood pressure in adolescent offspring of diabetic mothers. J Pediatr. 2000;136(5):587-592.

16. Grunnet LG, Hansen S, Hjort L, et al. Adiposity, dysmetabolic traits, and earlier onset of female puberty in adolescent offspring of women with gestational diabetes mellitus: a clinical study within the Danish National Birth Cohort. Diabetes Care. 2017;40(12):1746-1755.

17. Lee JH, Kim HC, Kang DR, Suh I. The 23-year tracking of blood lipids from adolescence to adulthood in Korea: the Kangwha study. Lipids Health Dis. 2017;16(1):221.

18. Clarke WR, Schrott HG, Leaverton PE, Connor WE, Lauer RM. Tracking of blood lipids and blood pressures in school age children: the Muscatine study. Circulation. 1978;58(4):626-634.

19. Chen X, Wang Y. Tracking of blood pressure from childhood to adulthood: a systematic review and meta-regression analysis. Circulation. 2008;117(25):3171-3180.

20. Webber LS, Srinivasan SR, Wattigney WA, Berenson GS. Tracking of serum lipids and lipoproteins from childhood to adulthood. The Bogalusa Heart study. Am J Epidemiol. 1991;133(9):884-899.

21. Rademacher ER, Jacobs DR Jr, Moran A, Steinberger J, Prineas RJ, Sinaiko A. Relation of blood pressure and body mass index during childhood to cardiovascular risk factor levels in young adults. J Hypertens. 2009;27(9):1766-1774.

22. de Zegher F, Devlieger H, Eeckels R. Fetal growth: boys before girls. Horm Res. 1999;51(5):258-259.

23. Clifton VL. Review: sex and the human placenta: mediating differential strategies of fetal growth and survival. Placenta. 2010;(31 Suppl): S33-S39.
24. Eriksson JG, Kajantie E, Osmond C, Thornburg K, Barker DJ. Boys live dangerously in the womb. Am J Hum Biol. 2010;22(3):330-335.

25. Perng W, Rifas-Shiman SL, Hivert MF, Chavarro JE, Sordillo J, Oken E. Metabolic trajectories across early adolescence: differences by sex, weight, pubertal status and race/ethnicity. Ann Hum Biol. 2019;1-10.

26. Classification and diagnosis of diabetes mellitus and other categories of glucose intolerance. National Diabetes Data Group. Diabetes. 1979;28(12):1039-1057.

27. Barker DJ, Bagby SP. Developmental antecedents of cardiovascular disease: a historical perspective. J Am Soc Nephrol. 2005;16(9):25372544.

28. de Onis M, Onyango AW, Borghi E, Siyam A, Nishida C, Siekmann J. Development of a WHO growth reference for school-aged children and adolescents. Bull World Health Organ. 2007;85(9):660-667.

29. Weston AT, Petosa R, Pate RR. Validation of an instrument for measurement of physical activity in youth. Med Sci Sports Exerc. 1997;29 (1):138-143.

30. Cullen KW, Watson K, Zakeri I. Relative reliability and validity of the block kids questionnaire among youth aged 10 to 17 years. J Am Diet Assoc. 2008;108(5):862-866.

31. Perng W, Villamor E, Mora-Plazas M, Marin C, Baylin A. Alphalinolenic acid (ALA) is inversely related to development of adiposity in school-age children. Eur J Clin Nutr. 2015;69(2):167-172.

32. Perng W, Mora-Plazas M, Marin C, Villamor E. Iron status and linear growth: a prospective study in school-age children. Eur J Clin Nutr. 2013;67(6):646-651.

33. Perng W, Mora-Plazas M, Marin C, Rozek LS, Baylin A, Villamor E. A prospective study of LINE-1DNA methylation and development of adiposity in school-age children. PLoS One. 2013;8(4):e62587.

34. Regnault N, Kleinman KP, Rifas-Shiman SL, Langenberg C, Lipshultz SE, Gillman MW. Components of height and blood pressure in childhood. Int J Epidemiol. 2014;43(1):149-159.

35. U.S. Department of Health and Human Services. The fourth report on the diagnosis, evaluation, and treatment of high blood pressure in children and adolescents. 2005.

36. Weissgerber TL, Mudd LM. Preeclampsia and diabetes. Curr Diab Rep. 2015;15(3):9.

37. Crispi F, Miranda J, Gratacós E. Long-term cardiovascular consequences of fetal growth restriction: biology, clinical implications, and opportunities for prevention of adult disease. Am J Obstetr Gynecol. 2018;218(2):S869-S879.

38. Mercuro G, Bassareo PP, Flore G, et al. Prematurity and low weight at birth as new conditions predisposing to an increased cardiovascular risk. Eur J Prev Cardiol. 2013;20(2):357-367.

39. Kerkhof GF, Breukhoven PE, Leunissen RW, Willemsen RH, HokkenKoelega AC. Does preterm birth influence cardiovascular risk in early adulthood? J Pediatr. 2012;161(3):390-6.e1.

40. Wilk M, Horodnicka-Jozwa A, Moleda P, Petriczko E, Safranow K, Walczak M. Assessment of selected lipid parameters in in children exposed to gestational diabetes (GDM) in utero. Pediatr Endocrinol Diabetes Metab. 2016;22(4):140-147.

41. Ryckman K, Spracklen C, Smith C, Robinson J, Saftlas A. Maternal lipid levels during pregnancy and gestational diabetes: a systematic review and meta-analysis. BJOG. 2015;122(5):643-651.

42. Talton OO, Bates K, Salazar SR, Ji T, Schulz LC. Lean maternal hyperglycemia alters offspring lipid metabolism and susceptibility to dietinduced obesity in micedagger. Biol Reprod. 2019;100(5):1356-1369.

43. Alfaradhi MZ, Fernandez-Twinn DS, Martin-Gronert MS, Musial B, Fowden A, Ozanne SE. Oxidative stress and altered lipid homeostasis in the programming of offspring fatty liver by maternal obesity. Am J Physiol Regul Integr Comp Physiol. 2014;307(1):R26-R34.

44. Cook S, Auinger P, Huang TT. Growth curves for cardio-metabolic risk factors in children and adolescents. J Pediatr. 2009;155(3):S6.e15S6.e26. 
45. Amri K, Freund N, Vilar J, Merlet-Benichou C, Lelievre-Pegorier M. Adverse effects of hyperglycemia on kidney development in rats: in vivo and in vitro studies. Diabetes. 1999;48(11):2240-2245.

46. Nelson RG, Morgenstern H, Bennett PH. Intrauterine diabetes exposure and the risk of renal disease in diabetic Pima Indians. Diabetes. 1998;47(9):1489-1493.

47. Schooling CM. Life course epidemiology: recognising the importance of puberty. J Epidemiol Community Health. 2015;69(8):820.

48. Raitakari OT, Juonala M, Kahonen M, et al. Cardiovascular risk factors in childhood and carotid artery intima-media thickness in adulthood: the cardiovascular risk in young Finns study. JAMA. 2003;290(17):2277-2283.

49. Camhi SM, Katzmarzyk PT, Broyles S, et al. Predicting adult body mass index-specific metabolic risk from childhood. Metab Syndr Relat Disord. 2010;8(2):165-172.

\section{SUPPORTING INFORMATION}

Additional supporting information may be found online in the Supporting Information section at the end of this article.

How to cite this article: Perng W, Hockett CW, Sauder KA, Dabelea D. In utero exposure to gestational diabetes mellitus and cardiovascular risk factors in youth: A longitudinal analysis in the EPOCH cohort. Pediatric Obesity. 2020;15:e12611. https://doi.org/10.1111/ijpo.12611 